Bolyai Society - Springer-Verlag

\title{
ERRATUM
}

\section{ALMOST COVERS OF 2-ARC TRANSITIVE GRAPHS*}

\author{
SANMING ZHOU
}

Received January 22, 2007

In Theorem 3.1 the integer $n \geq 4$ is not necessarily even, and the last sentence of the first paragraph on p. 740 should be deleted or replaced by the following explanation. (This change affects neither the rest of the proof of Theorem 3.1 nor the proof of other results in the paper.)

"Thus, if $n$ is even, then consecutive terms in the sequence $\sigma_{0} \sigma_{1}, \sigma_{1} \sigma_{0}$, $\sigma_{2} \sigma_{3}, \sigma_{3} \sigma_{2}, \ldots, \sigma_{n-2} \sigma_{n-1}, \sigma_{n-1} \sigma_{n-2}, \sigma_{0} \sigma_{1}$ are non-adjacent and adjacent in $\Gamma$ alternatively, and consecutive terms in $\sigma_{1} \sigma_{2}, \sigma_{2} \sigma_{1}, \sigma_{3} \sigma_{4}, \sigma_{4} \sigma_{3}, \ldots, \sigma_{n-1} \sigma_{0}$, $\sigma_{0} \sigma_{n-1}, \sigma_{1} \sigma_{2}$ are non-adjacent and adjacent in $\Gamma$ alternatively. If $n$ is odd, then consecutive terms in the sequence $\sigma_{0} \sigma_{1}, \sigma_{1} \sigma_{0}, \sigma_{2} \sigma_{3}, \sigma_{3} \sigma_{2}, \ldots, \sigma_{n-1} \sigma_{0}$, $\sigma_{0} \sigma_{n-1}, \sigma_{1} \sigma_{2}, \sigma_{2} \sigma_{1}, \ldots, \sigma_{n-2} \sigma_{n-1}, \sigma_{n-1} \sigma_{n-2}, \sigma_{0} \sigma_{1}$ are non-adjacent and adjacent in $\Gamma$ alternatively."

Accordingly, in Theorem 1.1, Proposition 3.4, Corollary 4.1, Corollary 4.2(b) and the abstract of the paper, the integer $n \geq 4$ is not necessarily even. The word "even" should also be removed from the following locations: p. 740 , line 27 ; p. 741 , line 6 from the bottom; p. 742, line 4; and p. 743, line 4 from the bottom.

The following example (due to an anonymous referee for another paper of the author) shows that $n$ can be an odd integer indeed. The Petersen graph $\Sigma$ can be defined as having vertices the unordered pairs $i j$ of distinct elements of $\{1,2,3,4,5\}$ such that $i j$ and $i^{\prime} j^{\prime}$ are adjacent if and only if $\{i, j\} \cap\left\{i^{\prime}, j^{\prime}\right\}=\emptyset$. The alternating group $G:=A_{5}$ induces a 2 -arc transitive group of automorphisms of $\Sigma$, and $\Delta:=(35,12,34,51)^{G}$ is a self-paired $G$ -

Mathematics Subject Classification (2000): 05C25, 05E99

* Combinatorica 24(4) (2004), 731-745 
orbit on $A_{3}(\Sigma)$. It can be verified that the 3 -arc graph $\Gamma:=\operatorname{Arc}_{\Delta}(\Sigma)$ is an almost cover of $\Sigma$, and that $\Sigma$ is a near 5-gonal graph with respect to the $G$-orbit $\mathcal{E}:=(12,34,51,24,35,12)^{G}$ on 5 -cycles of $\Sigma$.

Sanming Zhou

Department of Mathematics and Statistics

The University of Melbourne

Parkville, VIC 3010

Australia

smzhou@ms . unimelb.edu . au 